is out of place in the civilian clinic, and vice versa. A large measure of revision of our standard books, however, will be necessary in the light of the experience of the war.

As bearing alike on methods of amputation and the control of haemorrhage the femoral artery, the largest peripheral trunk in the body, presents some problems in operative surgery the interest of which has a wider range than the anatomical distribution of that artery. Amongst these problems the following are of constant recurrence:

\section{The Control of Haemorrhage during the Operation} of Amputation at the Hip-joint.

Amputation at the hip (transarticular, transcervical, transtrochanteric, or subtrochanteric) is one of the most common and one of the most critical of amputations. Haemorrhage is its chief risk. Our standard works contain descriptions of various methods of controlling it, each of which has its advocates. 'In the interesting work, Amputation Stumps, recently issued by G. Martin Huggins, the author, in discussing amputation at the hip, warmly advocates a variety of the operation well known as the "anterior racket" with ligature of the femoral vessels as the first step. Mr. Huggins, evidently in view of much of the teaching of the textbooks, describes his operation as "unorthodox." If it be so, one cannot but think that his heterodoxy will find support. Indeed, so far as the control of haemorrhage is concerned, some surgeons have long been converts to the method, and the experience of operating under war conditions is adding to their number. For years I have practised no other method, and in the printed notes on operative surgery issued to my classes have indicated its advantages.

Work during the war has confirmed the favourable opinion of the method. Initial ligation of the femoral vessels renders the surgeon independent of skilled assist. ance, and removes all risk of serious loss of blood, for the only other vessels severed in the operation are compara. tively smail branches of the sciatic and gluteal arteries. The method has had its supporters for years during peace times. Mr. Huggins, with exceptional experience of war surgery, commends it as the method of choice. In the light-both of pre-war and war experience, many surgeons, of whom I am one, advocate it as the best method. It is evident that its adoption as such would necessitate the relegation of the textbook descriptions of not a few classic methods to the records of the past. Surgeons will watch with interest the decision finally arrived at, the more so that the principle involved in the initial ligation of the main vessels at the site of amputation is applicable to other amputations than that at the hip.

The Control of Haemrorrleage in Amputation of any Portion of the Lower Limb Below the Upper Third of the Thigh.

In such amputations, and in operations for compound "smashed" bones, haemorrhage may be contiolled by one or other of three methods-namely, the tourniquet, manual compression of the femoral artery, and temporary ligation of that vessel. The last mentioned, however useful it may be in a few cases in civil practice, has little field in war surgery, where the choice lies between the tourniquet on the thigh and digital compression of the femoral artery. 'The tourniquet is necessarily in universal use at present at the fighting fronts, where there is ro substitute for it in certain circumstances. Its use also in operations is part of the stock teaching of our textboolss. But opinion is veering against its use. In the last issue (1918) of the official Manual of Injuries and Diseases of War it is stated that:

The systematic use of the elastic tourniquet cannot be too severely condemned. The employment of it, except as $n$ temporary measure during an operation, usually indicates that the person employing it is quite ignorant both of how to stop bleeding properly and also of the danger to life and limb caused by the tourniquet.

And again :

Even if applied only for an hour it greatly interferes with the nutrition of the wounded tissues, and so favours the development of ianaërobic organisms.

Thatiand much of the teaching of our textbooks, how. ever, still leaves the tourniquet, in spite of its dangers and disadrantages, apparently as : the routine method of controlling haemorrhage during amputations and other opera- tions on the limbs. Its disappearance from the operating theatre would involve considerable revision of sections of our textbooks. Opinion hias not yét crystallized, but there are many surgeons who at present very rarely indeed use a tourniquet, and that only when amputating with no assistance, skilled or unskilled. I have not for many years used a tourniquet for any operation, and have found, in the lower limb, digital compression of the femoral artery against the pubic ramus quite efficient. A colleague requires no instruction, but an orderly, a sister, or a student, if acting for the first time, generally requires coaching on three points-namely, to stand on the outer side of the limb, to fix the thumbs for counter-pressure on the postero-external aspect of the ilium below the level of the anterior superior spine, and to press the fingers on the vessel just below Poupart's ligament. If the fingers are placed lower down the limb the artery is not caught firmly against the pubic ramus, and if the thumbs are placed against the trochanter instead of the ilium the hands are apt to be displaced during the operator's manipulations of the limb. Digital compression of the femoral is quite easily maintained by any hand of average size and strength in even the most muscular patient during the course of any amputation. During such operations as that for removal of smashed bone and damaged tissue or the excision of a tumour, compression is of ten not required at all, the compresser simply lieeping his fingers on the femoral pulse ready to tighten his grasp instantly ou request.

\section{Prevention of Secondary Haemorrhage in "Open"} Amputation Stumps in the Thigh.

Open stumps include guillotine stumps, flap stumps awaiting secondary suture, and stumps in which the sutures have cut out and the wound is gaping. The last is always, and the two first named ultimately often, suppurating and sloughing. Under such conditions secondary haemorihage is a constant risk at any date after the first week, by which time the ligature is absorbed or dissolved in the pus. In all open septic stumps in the thigh which are a week or more old I make. it a routine practice, as a preventive measure, to ligature the common femoral vessels. By the end of the first week after amputation the collateral circulation after ligature of the common femorals is ample for the needs of any stump down to the supracondylar level. For open stumps below that level, and certainly for those below the knee, ligature of the femoral in a septic limb carries a grave risls of resulting gangrene. Fortunately the need for it in such cases is less clamant than in thigh stumps. In the latter secondary haemorrhage constitutes too often a rapidly completed tragedy. In leg stumps the flow is much less copious (from one only of the three branches, anterior or posterior tibial or peroneai) and leaves time for the sister or orderly to apply the tourniquet or compress the femoral while the assistance of the surgeon is being got.

\section{THE CURSE OF IMIOBILIZATION.} BY

\section{J. W. DOWDEN, M.B., C.MI., F.R.C.S.Edin.,} SURGEON TO THE ROYAL INFIRAARY, WDINBURGH.

Thousanos of our soldiers have been more or less disabled for life simply and solely on account of prolonged immobilization of injured limbs. Case after case could be cited as examples of the harm done, and I an sure many readers of this Journal can endorse the statement.

I have had four year's' experience of war surgery in this country, first in a Territorial general hospital for a year, and for the last three year's in a war hospital where I had between two and three hundred beds rander my charge; besides this I have seen many cases in the Edinburgh Royal Infirmary and in private practice. I may therefore claim to have some experience.

If asked as to the lessons learnt during those four years, I would emphatically say, first, that nature is the most wonderful surgeon, especially when enconraged; secondly, that septic wounds should be thoroughly drained; thirdly, that from the very beginning frequent passive and active movements should be carried ont; steadily increasing the range; that splints should never be used except when 
absolutely necessary, and then only for as short a time as possible, and should never interfere with passive and active movements. I am writing now mainly of the upper extremity, but I am not by any means excluding the lower. Massage is a most excellent adjunct-far and away the best-to active movement. I lay but little stress on electricity or baths.

In war surgery the future usefulness of the wounded must be borne in mind, and in that relation the order of importance is-muscles, joints, and bones; not bones, joints, muscles. If a demand is made upon nature without unclue pain, and carefully advancing, nature will always respond. Disuse, active and passive, means malnutrition of the part. Muscles atrophy, bones atrophy, blood vessels atrophy, pus tends to track along the intermuscular planes; passive and active movement help to drive the pus out.

When passive or active movements are begun early, pain is the danger signal that shows harm is being done, and the movements should be modified temporarily. When after prolonged immobilization adhesions have formed, however, pain has to be endured while the adhesions are being stretched or broken. I am not at all surprised, therefore, that there has been an insistent demand for the services of bonesetters, who overcome the adhesions which have been brought about by well meaning surgeons. Take, for example, the case of a man shot through the biceps. His forearm was placed at a right angle and kept in that position for weeks; he was then unable to extend the arm, and after many long weeks of massage recovery was incomplete. In the case of wounds through the forearm where the bones and nerves have escaped, and treated with splints for prolonged periods, it is found that when movements of a passive and active nature are attempted the patient is unable to carry them out owing to the formation of fibrous tissue during the period of immobilization. The most marked results from muscles involved are found in the hamstring muscles and the calf. How familiar is the sight of convalescent soldiers limping about the streets with bent, knees and on their toes! The majority of these will never straighten their legs again or dorsiflex the foot in walking, owing entirely to neglect of the muscles and joints, and constant straightening and correcting by active and passive movements. Injuries to joints, even though septic, are not a bar to movement; the joints should be gently moved and encouraged, short of pain, and it is wonderful what results will ultimately be obtained, provided attention is paid daily to movement (passive at tirst and later active and steadily increased).

As to pain, the attitude of most present-day surgeons is wrong. For the last twelve years I have treated all frac. tures of the scapula, clavicle, liumerus, and many of the forearm, withont splints. I have no hesitation in applying splints if I think they are needed, but after the thircl or fourth day patients are satisfied with the lie of their arms in a sling. 'The main point, however, is that every movement of all joints and muscles should be encouraged, first of all passively and as soon as possible actively, and this should be carried out frequently during the day. It is sur. prising to see a patient with a fractured humerus, with possibly overlapping, extending the arm, flexing it and lifting it from the side, in the course of a fortnight or three weelss; by the time the fracture has consolidated all the movements of the joints are perfect, provided the damage has not been too great. In simple fractures the results are admirable; in septic compound fractures I can say the same; but.watch must be kept for collections of pus about necrotic bone, and these must be opened and drained, but should never interfere with the passive and active movements of the part. I feel compelled to write this, because 1 have seen such bad results not only from hospitals abroad but from hospitals in England and scotland. A few examples of many that have passed through my hands may perhaps help to impress my view.

(a) Through-and-throngh bullet wound of the hamstring, with flexion of the knee, kept in the position of flexion for several weeks before coming under my care. Twenty to thirty pounds pressure over the extended knee cannot overcome the contraction, and the man is a probable cripple for life, walking on his toes with bent knee.

(b) Patient with a wound of the outer side of the humerus not completely fracturing the bone, kept in a right angle splint for eight weeks. Unable to flex and extend elbow. rigidity about the shoulder, unable to satisfactorily close the haud; now, after four months of massacse, unable to straighten the arm or flex it completely.

(c) Patient with a suppurating prepatella bursa kept for three weeks in a posterior splint and three weeks in a Thomas splint. Now scarcely any flexion of the knee.

(d) Patient with a suppurating and ankylosing knee-joint several months in splints. No movement in the plantar flexed toes, plantar flexion of the foot, and no dorsitlexion of the foot possible.

(e) Numerous fractures of the homerus kept three and four months in a right-angle splint, hecause there was a septic wound leading to bone. Practically no movement at shoulder, elbow, wrist, and, in some cases, fingers.

I could go on giving endless cases of a similar nature. This needless disablement of men is really the most dis. heartening thing I know in the war. Sir Auckland Geddes as a medical man, and certainly the Minister of Pensions, should look into the matter with care, for it is one which involves the man power of the nation. I feel absolutely certain that the future will see a great reaction from this most remarkable age of splints.

\section{VARIATIONS IN THE ACTIVITY OF THE CILIARY MUSCLES.}

BY

\section{MAJOR G. F. ALEXANDER, R.A.M.C.}

THE activity of the ciliary muscles can be realized only by the routine practice of estimating the refraction when they have been completely paralysed, and again after they have recovered from the effects of the cycloplegic. Complete paralysis being litherto only obtainable by the use of atropin, has been mainly restricted to children on account of the inconvenience of the prolonged paralysis of accommodation and the danger of inducing glaucoma in the elderly, and thus, as a 2 per cent. solution of homatropiu and cocaine has in the case of young and vigorous adults frequently only a partial paralysing effect, variations in the activity of the ciliary muscles in adults have largely escaped observation. Early in the year, however, I found that cycloplegia as complete as that obtained by atropin could be obtained by a saturated solution of homatropin (16. per cent.); a large drop may be instilled in each eye without fear of toxaemia, and recovery is as rapid as after. the use of the 2 per cent. solution-that is, in twenty-four to thirty-six hours-or if a drop of 1 per cent. eserin is instilled in two to three hours, and thus the benefit of atropin is obtained without the drawback of its prolonged paralysing effect. By the use of this solution in over 1,000 cases, supplemented by that of a special astigmatic chart, I have been able to observe some interesting variations in the activity of the ciliary muscles, which with those alreacly familiar are briefly summarized as follows:

1. Both in hypermetropia and myopia the tonicity of the ciliary muscles varies from time to time: the difference in the vision recorded by different observers or the same observer on different days is thus accounted for, increase of activity necessarily lessening hypermetropia and in creasing myopia, and relaxation the reverse.

2. In anisometropia unequal activity of the ciliary muscles frequently neutralizes more or less of the disparity.

3. Apart from anisometropia the ciliary muscles often show unequal tonicity, as is proved-(a) in either hypermetropia or myopia by difference in the corrections of the two eyes found on post-cycloplegic testing, although paralysis of the muscles showed that the eyes were equaliy ametropic; (b) in either hypermetropia or myopia, and even occasionally in emmetropia, by spasm of the accon. modation taking place to an unequal degree in the two eyes, and even occasionally in one eye only.

4. Spasm of accommodation, apart from its commonest causes-namely, astigmatism and exophoria, the latter naturally acting chiefly in myopia-is frequent from overuse of the eyes for near work, especially if tine, under the electric light, or in insufficient light: though naturally most marked in hypermetropia it is found also in myopia and emmetropia; it is observed chiefly in young and neurotic subjects, but is found also in others, and even when they are well beyond the presbyopic age (I have notes of one case of spasm in an enmetrope aged 48 year's). 